\title{
Aproximación al uso de fuentes documentales primarias en la ESO. Una propuesta didáctica a partir de la crisis económica de 1917 en Málaga
}

Approach to the use of primary documentary sources in the ESO. A didactic proposal based on the economic crisis of 1917 in Malaga

Belén Calderón Roca $\left(^{*}\right)$

\section{Resumen}

El estudio de la historia local partiendo la crisis española de 1917 en Málaga, pretende ofrecer unas pautas didácticas para adiestrar al alumnado de $3^{\circ}$ de la ESO, en el conocimiento y uso de las fuentes documentales que han posibilitado el estudio de un tema historico. Bajo la tutela del doc ente, el al umnado t rabajará des de I a bi bliografía gener al a I a es pecífica l ocal, par a posteriormente r ealizar u $\mathrm{n}$ rastreo ar chivístico y el vaciado d ocumental de un archivo local, aprendiendo a realizar búsquedas de fuentes documentales por diferentes cauces, digitales y físicos, y a $r$ ealizar $f$ ichas $c$ on I a documentación consultada. E llo I e pe rmitirá ad quirir contenidos, $\mathrm{m}$ ediante I a m anipulación de doc umentos gener ados en otra é poca y circunstancias ex trapolables al $\mathrm{pr}$ esente... $\mathrm{P}$ ero además, tomar contacto $\mathrm{d}$ irecto $\mathrm{c}$ on $\mathrm{e} \mathrm{I}$ patrimonio cultural local, en definitiva, que podrá tocar, leer y oler.

Palabras Clave: Aprendizaje de la historia, Currículo ESO, Fuentes documentales primarias, Crisis económica, Málaga.

\begin{abstract}
The study of local history starting from the Spanish crisis of 1917 in Malaga, aims to provide didactic gu idelines t o $t$ rain s tudents in the $3 r d$ year of E SO, in the k nowledge and us e of documentary sources that have enabled the study of a historical topic. Under the guidance of the $t$ eacher, $t$ he $s$ tudents will work $f$ rom $t$ he gen eral bibliography $t$ ot he specific I ocal bibliography, to later carry out an archival search and the document draining of a local archive, learning to search for documentary sources through different channels, digital and physical, and to $m$ ake catalographic $f$ iles with the consulted doc umentation. This will allow you to acquire content, through the manipulation of documents generated in another time and circumstances extrapolated t o the pr esent... But a Iso... to $\mathrm{c}$ ome into direct $\mathrm{c}$ ontact with $\mathrm{t}$ he I ocal $\mathrm{c}$ ultural heritage, in short, that will be able to touch, read and smell.
\end{abstract}

Key words: History learning, curriculum, Primary documentary sources, Economic crisis, Malaga.

\footnotetext{
* Universidad de Málaga,. belencalderon@uma.es ORCID 0000-0003-0317-2548
}

Calderón Roca., B. (2021). Aproximación al uso de fuentes documentales primarias en ESO. Una propuesta didáctica a partir de la crisis de 1917 en Málaga. CLIO. History and History teaching, 47, 276-294. https://doi.org/10.26754/ojs clio/clio.2021476148 Recibido: 30/6/2021. Aceptado: 3/12/2021. 


\section{Introducción. De lo local a lo global mediante el estudio del marco cronológico-espacial.}

España se vio af ectada ent re 1914 y 1923 po r una se rie de pr oblemas de índole heterogénea (ruptura del liberalismo español, descomposición del si stema pol ítico canovista, o co nsecuencias económicas derivadas de la primera guerra mundial), que abrieron vía a las primeras movilizaciones obreras y revueltas sociales, que caracterizaron a la grave crisis social, económica e ideológica de 1917. La neutralidad española derivó en la exportación incontrolada de bienes de consumo, lo que originó una gr avísima cr isis de su bsistencias, ade más del incremento de la ca restía en productos de primera necesidad, con precios disparados, lo que se manifestó de inmediato en el descontento de I a población. A la conmoción social, provocada por diversos sectores militares y burgueses, se unieron grupos de proletariado, por lo que la conflictividad y las protestas se incrementaron ca da vez más. Había arraigado la "cristalización de una co nciencia obrera en todo el país" (Ramos, 1987, p. 188) y ello preparó el terreno para la protesta general del día 18 de diciembre de 1916. Al caciquismo, el desg obierno y el nepot ismo, así co mo la i ncapacidad d e l levar a I a práctica eficiente planes de reformas sociales y económicas desde los poderes públicos, se unió la impopularidad de I os países que participaban en I a guerra y las medidas represivas contra la población. Ésta se lamentaba y se revolvía, y ello se refleja magníficamente en las portadas de los periódicos locales de la época, como, por ejemplo, El Popular o El Regional, ambos de Málaga.

Sin embargo, y así lo afirma Tuñón de Lara (1974), en Andalucía la huelga no prosperó como en ot ras regiones de E spaña, sa lvo en I as cuencas mineras de Li nares, La Carolina y Río Tinto, y algunbos conatos de huelga parcial producidos en Córdoba y Jerez. En Málaga la huelga estalló el día 16 de agosto y continuó un día más, aunque ya se enco ntraba vencida en sus núcleos principales, por lo que las repercusiones fueron muy desiguales. Por el contrario, la huelga ferroviaria obtuvo éxito total y a ellos se unieron los conductores de algunas agencias de transportes, operarios de fábricas de harinas y de la empresa de productos químicos Colores Minerales. Por el contrario, no prosperó en la Pesquera Malagueña, ni en los Altos Hornos. En cualquier caso, se cuentan 350 obreros concentrados en núcleos centrales de la ciudad como laPlaza de Arriola, el $\mathrm{P}$ asillo de $\mathrm{S}$ anta Isabel y en I as calles adyacentes; trabajadores que 
reclamaban el aumento de sus salarios y la disminución de su jornada laboral.

Durante la huelga, los dispositivos de vigilancia se incrementaron, y ello se refleja en la actitud del Gobernador Militar, que puso en práctica un impresionante despliegue policial, en pr evisión de posi bles altercados, pues incluso se practicaron numerosas detenciones. Tras la huelga, sin embargo, todas las fuentes consultadas coinciden en destacar la act itud pacífica de I os huel guistas, tal y co mo indica $\mathrm{M}^{\mathrm{a}}$ Dolores Ramos (1987). Es reseñable que la alta burguesía malagueña elogió tanto el buen desarrollo del conflicto, como la conducta llevada a cabo por las autoridades, aunque en algunos periódicos como El Regional, se condenó públicamente a los huelguistas, que continuaron con las movilizaciones de masas tras el conflicto.

Desde 1917 el estado de excepción en España se convirtió en un hecho generalizado. Los mecanismos de poder de las oligarquías y sectores de la alta burguesía española ejercieron una fuerte presión y trascendieron sobre las instituciones del Estado, subordinando el interés colectivo a sus fines particulares.

\section{Objetivos y metodología.}

\subsection{Objetivos.}

El estudio de la historia local mediante la crisis española de 1917 en M álaga pretende ofrecer unas pautas didácticas para que el alumnado entienda que conocer el pasado resulta i ndispensable $p$ ara interpretar el presente, a sí co mo par a co municarse y convivir democráticamente en el futuro. Mediante la coeducación y trabajo cooperativo, estudiando episodios relevantes como las repercusiones de la huelga general del 13 de agosto de 1917 como punto de partida, podremos introducir al alumnado de $3^{\circ}$ de la ESO, en el conocimiento y uso de las fuentes documentales que han posi bilitado el estudio del tema (según determina la Orden de 15 de ene ro de 2021 por la que se desarrolla el currículo correspondiente a la etapa de Educación Secundaria Obligatoria en la Comunidad Autónoma de Andalucía). Bajo la tutela del docente, el alumnado trabajará desd e I a bi bliografía general a I a esp ecífica I ocal, para post eriormente realizar un $r$ astreo a rchivístico y vaciado do cumental de a rchivos malagueños, 
aprendiendo a r ealizar búsquedas de fuentes documentales y a realizar fichas con la documentación consultada.

No se trata de co nfeccionar m ediante est e trabajo una hi storia local de I a crisis en Málaga durante esta fecha, de hecho parte de nuestro título "aproximación al uso de fuentes documentales primarias", procuramos ofrecer, $p$ recisamente, pautas al alumnado para aproximarse al conocimiento de las repercusiones de la huelga general tenida lugar el 13 de agosto de 1917 en Málaga. Con ello, pretendemos introducirles en el uso de las fuentes documentales que han posibilitado su estudio, y que permitirán, a posteriori, realizar revisiones de los mismos o efectuar nuevas aportaciones. $\mathrm{P}$ orque I a h istoria se $\mathrm{r}$ epite, c on I o cu al, a través de este trabajo, eminentemente práctico, efectuaremos junto al alumnado un recorrido iniciado desde la bibliografía más general, buscando menciones a nuestra capital, para posteriormente, introducirnos en internet y enseñarles a buscar, escrutar y discriminar la información, para ocuparnos de aquella relevante sobre episodios concretos en el ámbito local.

\subsection{Metodología}

Hemos estructurado el trabajo que deberán realizar los alumnos en 4 fases:

- Fase 1: Búsqueda en internet.

-Fase 2: $V$ aciado bi bliográfico y docu mental en bases de datos, $h$ emerotecas y bibliotecas universitarias y científicas (RECOLECTA, DIALNET, EUROPEANA y el CATÁLOGO JÁBEGA (Biblioteca de la Universidad de Málaga) y ARCHIVO DIGITAL DÍAZ DE ESCOVAR DE MÁLAGA (ADDEM).

-Fase 3: Rastreo archivístico de aproximación: PARES.

-Fase 4: Rastreo y vaciado documental del ARCHIVO HISTÓRICO MUNICIPAL DE MÁLAGA (AHMMA).

\subsubsection{Fase 1: Búsqueda en internet.}

Se efectuará una búsqueda preliminar en red a través de los buscadores generalistas más utilizados, como son Google, Bing o Yahoo, así como a través de otros más 
especializados como Google Académico. Al realizar una búsqueda básica en Google con la pal abra cl ave: "crisis malaga 1917", en I a p rimera página pudi mos encontrar los siguientes resultados. Aparte del ruido ${ }^{1}$ entremezclado con los resultados realmente i nteresantes par a nuestro $t$ rabajo, los tres primeros resultados que aparecieron nos remitían a webs de librerías donde se vendían títulos relacionados con el aco ntecimiento (Casa del libro y Fnac), en co ncreto de I a aut ora $\mathrm{M}^{\mathrm{a}} \mathrm{D}$ olores Ramos. El siguiente artículo fue un enlace a Dialnet donde se alojaba un artículo de la misma autora.

Los siguientes resultados arrojados por Google repetían enlaces a librerías con idénticos títulos, pero el último resultado fue un enlace a la Wikipedia co n el título "Crisis española de 1917": https://es.wikipedia.org/wiki/Crisis espa\%C3\% B1ola de 1917. En esta web de acceso libre, como sabemos, se muestra información que puede ofrecer datos muy básicos, o por el contrario, exhaustivos, dependiendo de quien la incorpore mediante registro previo. Por ello hay que enseñar a los alumnos a ser cautelosos en la ut ilización de I a misma y confrontar es ta fuente digital co n ot ra información. Los datos que ofrecía la wiki eran su cintos pero bien estructurados en bloques: 1) Coyuntura internacional, 2) La crisis en España, 3) Referencias , 4) Véase también y 5) Enlaces externos. En el apartado de bibliografía se citaban varios títulos, aunque algunos de ellos eran muy básicos y las referencias omitían cualquier mención a obras locales.

En la segunda página, el primer resultado nos condujo a la web institucional del Ayuntamiento de Málaga:

http://www.malaga.eu/ayto/m ciudad/portal/menu/seccion 0005/secciones/subSeccion 0007. En un apar tado m uy so mero so bre e femérides malagueñas, se m encionaba este epi sodio, agudizando I a co nflictividad so cial q ue pr odujo di cha $\mathrm{cr}$ isis revolucionaria. E I cu arto r esultado co ndujo a un $\mathrm{t}$ rabajo ci entífico en $\mathrm{P}$ DF de 55 páginas titulado: "Huelva y la crisis de 1917", donde se mencionaba de nuevo el libro de $M^{a}$ Dolores Ramos. $Y$ el quinto resultado nos llevó a la web de la Universidad de Málaga donde apar ecía I a ficha-cv de I a P rofa $D$ ra. $M{ }^{a} D$ olores Ramos Palomo, Catedrática de Historia Contemporánea y evidentemente, especialista en el tema.

\footnotetext{
${ }^{1}$ Se denomina así a la información no relevante para nuestro estudio encontrada durante la búsqueda.
} 
Sin em bargo, en Google A cadémico (Scholar Google) co n la misma palabra cl ave: "Málaga cr isis 1917" efectuamos búsqueda simple y av anzada, y aparecieron resultados que remitían a citas bibliográficas de títulos bastante significativos sobre el acontecimiento en Málaga, así como a uno de obligada consulta sobre el tema en España, el de Juan Antonio Lacomba. En otros buscadores como Yahoo o Bing, la información obtenida no difirió demasiado de la hallada a través de Google y Scholar Google.

\subsubsection{Fase 2: Vaciado bibliográfico y documental en bases de datos, hemerotecas y bibliotecas universitarias y científicas.}

Dentro de un mayor nivel de especialización, podríamos acudir (según el nivel y características del grupo escolar), en primer lugar, al uso de repositorios institucionales on-line co mo R ECOLECTA. E sta pl ataforma agr upa $t$ odos los repositorios de publicaciones científicas nacionales y permite acceder a documentación publicada en diferentes uni versidades sobre un t ema co ncreto. También $r$ esultan de par ticular relevancia los catálogos y bases de datos documentales como REBIUN, que aglutinan información reunida por bibliotecas universitarias españolas, públicas y privadas, así como por el C SIC. Y de su ma utilidad, so $\mathrm{n}$ ot ros repositorios de universidades españolas como DIALNET, e instituciones internacionales como EUROPEANA, que aglutinan diferentes tipos de documentación, así como INTERNET ARCHIVE DIGITAL LIBRARY, que alberga obras digitalizadas de prestigiosas bibliotecas como The Getty Research Institute, American Libraries, Canadian Libraries, University of Toronto Robarts Library, o California Digital Library, entre otros.

En lo que respecta a la búsqueda en RECOLECTA: http://recolecta.fecyt.es/recoleccion, esta plataforma no arrojó ningún resultado con las palabras clave "crisis malaga 1917", por ello cambiamos el criterio de búsqueda por las palabras clave: "malaga 1917" y "huelga 1917", ambos con cero resultados.

En cambio, con el criterio "crisis españa 1917" obtuvimos un único resultado referido al ámbito español, tratándose también de un texto de Juan Antonio Lacomba publicado en 1968.

Desde el repositorio REBIUN: http://www.rebiun.org/Paginas/Inicio.aspx, al introducir las palabras debúsqueda: "crisis 1917", encontramos 363 resultados. Algunos de ellos 
enlazaron co $\mathrm{n}$ t rabajos bast ante interesantes para co ntextualizar en $\mathrm{t}$ ema a ni vel estatal, y se recogen en las referencias bibliográficas de esre trabajo. Por otra parte, REBIUN of reció I a opc ión de el egir por $m$ aterias, y procedimos eligiendo: " crisis políticas España 1917" y “crisis económicas España 1917", co n lo que obt uvimos un único resultado, de nuevo el texto de obligada consulta de Lacomba (1970). En lo que respecta a EUROPEANA: http://www.europeana.eu/portal/es/collections/art-history y las palabras de búsqueda "crisis malaga 1917" no obtuvimos resultados.

En DIALNET: https://dialnet.unirioja.es/, nuestra búsqueda se orientó, esencialmente, a buscar y seleccionar aquellos títulos que estuvieran alojados on-line y proceder a su consulta. En el ca so de ot ros títulos sobre los que pudiéramos intuir interés para el tema, efectuamos una posterior búsqueda en catálogos de bibliotecas para -en caso necesario- acceder físicamente al texto. Hemos de decir que la búsqueda en Dialnet arrojó resultados bastante satisfactorios, especialmente, en lo que respecta a obras de carácter general y también sobre el ámbito específico de Málaga. Con el criterio de búsqueda: "crisis malaga 1917", obtuvimos dos resultados referidos a Málaga, siendo uno de el los la obra em blemática de I a Pra. Ramos: La crisis de 1917 en Málaga (1987), y curiosamente, otro de Lacomba sobre la crisis en Andalucía. Con el criterio "malaga 1917" encontramos doce artículos de revista, un artículo de libro y un libro. Y tras realizar una discriminación, únicamente nos sirvieron dos de ellos, ya mencionados. Por el c ontrario, co $\mathrm{nl}$ as palabras clave: " crisis españa 1917" , I os resultados fueron abrumadores, un total de dieciocho artículos de revista, cinco libros, cuatro a rtículos de $\mathrm{l}$ ibros y una $\mathrm{t}$ esis doctoral $q$ ue $\mathrm{v}$ ersaban so bre aspectos económicos y políticos relacionados con la crisis, destacando los que mencionamos en las referencias bibliográficas.

Con otras palabras clave "industria Malaga 1917" o "huelga España 1916" no obtuvimos ningún resultado. Sin embargo, con el criterio de búsqueda "huelga 1917" obtuvimos 24 resultados so bre las repercusiones de la huel ga en di versas ciudades como Madrid, Alicante, Navarra, Jerez de la Frontera o Vizcaya, entre otras. Asimismo, con las palabras clave: "Huelga 1916" obtuvimos nueve artículos de revista, además de un ar tículo y una t esis doctoral, d estacando el ar tículo de $\mathrm{M}{ }^{\text {a }} \mathrm{D}$ olores Ramos: "Conflictividad social en la coyuntura de la Primera Guerra Mundial: la huelga de Altos Hornos de Málaga (1916)". En una fase post erior, y $t$ ras los exiguos resultados 
encontrados en R EBIUN, pr ocedimos al $r$ astreo del ca tálogo bibliográfico de I a Universidad de Málaga (Catálogo Jábega): http://jabega.uma.es/ que consideramos de obligada consulta. Muchos de los títulos encontrados se repetían tras las otras búsquedas, por ejemplo los de $\mathrm{M}^{\mathrm{a}}$ Dolores Ramos, infinitamente repetidos; el de Jacinto Martín: Huelga general de 1917 (1971) o el de Juan Antonio Lacomba: La crisis española de 1917 (1970). No obstante, encontramos otro de éste úl timo autor, así como una obra general muy útil para contextualizar el tema de nuestro estudio.

Y para finalizar este epígrafe, efectuamos una consulta sobre prensa local de la fecha, en concreto de los diarios El Popular y El Regional, a través de la web del Archivo Díaz de Escovar de Málaga: http://www.museoartespopulares.com/archivo/, donde sus fondos se encuentran digitalizados. Hemos recogido en I as referencias bibliográficas de este trabajo dos títulos significativos.

\subsubsection{Fase 3: Rastreo archivístico de aproximación: PARES.}

En cuanto a fuentes documentales primarias, también hemos tenido en cuenta PARES (Portal de Archivos Españoles): http://pares.mcu.es., sitio que ofrece la información en sí, adem ás de dat os acerca de I a misma fuente que genera di cha do cumentación. Mediante búsquedas se ncillas o avanzadas, permite acce der a información sobre un tema específico y nos indica el centro documental donde se encuentra. Al acce der mediante el criterio de búsqueda sencilla con las palabras clave "crisis españa 1917", "crisis malaga 1917" e "industria malaga 1917" no se obt uvo ni ngún resultado. En cambio, I as palabras clave "huelga E spaña 1917", no s llevaron a I a S ección de I a Fiscalía del T ribunal S upremo, del A rchivo Hist órico Nacional ( $A H N$ ), dond e encontramos como título de I a uni dad docu mental: "Documentación de Fr ancisco Largo Caballero incorporada al procedimiento de la Causa General.", código de referencia: ES.28079.AHN/2.2.2.1.29.2.1, y fechas de creación: 1918 / 1937. Parte de la documentación de Francisco Largo Caballero (Madrid, 1869-París, 1946) se incorporó a la Causa General de la provincia de Madrid, (localizada en el paquete $\mathrm{n}^{\circ}$ 21 de apoyo a la Pieza Segunda del Alzamiento Nacional). Ello se debe a que en 1931 fue nombrado Ministro de Trabajo y Previsión Social del gobierno republicano de Manuel Azaña. Fue además un destacado dirigente obrero, de ahí su relación con la huelga de 1917, pues formó parte del Comité revolucionario que la organizó. Por ella fue det enido y co ndenado a ca dena per petua junto a ot ros de su s miembros. No 
obstante, en 1918 fue elegido diputado a las Cortes por Barcelona, siendo excarcelado y amnistiado, y e se mismo año, nombrado secretario general de la UGT, cargo que ocupó durante 20 años.

Por otra parte, en el Archivo del Centro Documental de la Memoria Histórica (ACDMH), encontramos la Sección: Delegación $\mathrm{N}$ acional de $\mathrm{S}$ ervicios Documentales de $\mathrm{I}$ Presidencia del G obierno, donde se custodia I a docu mentación p rocedente de I a incautación r elativa a Andrés Saborit C olomer (con t ítulo de I a unidad homónimo), código de referencia: ES.37274.CDMH/10.8.9.14.2584. Andrés Saborit Colomer (Madrid 1889 - Valencia 1980) fue tipógrafo y periodista, además de Presidente de las Juventudes Socialistas de E spaña y vicesecretario del $P$ artido $S$ ocialista $O$ brero Español desde 1915, ejerciendo en éste diversos cargos de responsabilidad, de ahí su vinculación con la huelga de 1917.

\subsubsection{Fase 4: Rastreo y vaciado documental archivístico: Relación de series y documentos del Archivo Histórico Municipal de Málaga.}

Tras realizar una breve visita al Archivo en grupo único, durante otra sesión accedimos con grupos de 10 al umnos a la Sala de Consulta. Preguntamos a los Jefes de sala y aceptamos sus sugerencias sobre la contextualización de nuestro t ema: crisis económica en Málaga y huelga de 1917, centrándonos en el rastreo las Actas Capitulares correspondientes al año 1916 y 1917. Efectuamos un escrupuloso vaciado documental de las páginas de sus libros, pues éstas se conservan encuadernadas, no en ca rpetas. Se trataba de enco ntrar información r elativa a fechas cl ave co mo: I a protesta general del 18 de diciembre de 1916, el manifiesto firmado por CNT y UGT el 27 de marzo de 1917 y la huelga general del 13 de agosto de 1917. Para ello, nos remitimos a la consulta de los meses previos y posteriores a las fechas en cuestión, estimando una franja de tres meses antes y tres meses después, de cada reunión del Cabildo Municipal de Málaga tenida en dichas fechas. Es decir, establecimos las siguientes franjas temporales en nuestra búsqueda:

-De septiembre a diciembre de 1916, y de enero a marzo de 1917.

-De mayo a noviembre de 1917. 
Entre la información en contrada so bre el tema (bastante exigua), se hace referencia entre otras cuestiones: a I a nece sidad de au tonomía de I os ayuntamientos, $m$ uy relacionada co n I a si tuación pol ítica que experimentaba E spaña en es a fecha; a I as concesiones a las industrias malagueñas, como por ejemplo, escrituras de propiedad por parte de la Comisión de Hacienda Municipal, o instalaciones e infraestructuras de otras empresas como la Compañía de gas o mataderos; se atienden asuntos relativos a los transportes, por ejemplo el se rvicio de bar co de v apor que enlazaba Málaga y Melilla, paralizado por Ia es casez de ca rbón; y también se tratan aspectos políticos como la manifestación pública de la impresión que causó el buen desarrollo de I as jornadas de la huelga en Málaga. La concreción de la información encontrada se anotó en cuadernos y luego se trabajaría en el aula, plasmándola en las fichas catalográficas en I as que se recogen: si clas del ar chivo del docu mento, fecha o fechas límite; signatura; Autor/es del documento; tipo de documento, breve resumen del contenido, tema, palabras clave y fotografía.

\section{Discusión y resultados.}

No hemos podido obtener aún resultados sobre esta propuesta didáctica que pensamos poner en marcha en I a pr axis, con nuestro al umnado d el Máster de Profesorado de Secundaria. Sin embargo, creemos indispensable discultir una serie de cuestiones con respecto a la ideoneidad de su planteamiento.

La generalización de las nuevas tecnologías ha añadido una nueva dimensión al uso de I as fuentes de ar chivo en el aul a, I o que ay uda a so Iventar g ran par te de I as dificultades de acceso a la documentación que antes existían. Sin embargo, todavía resulta indispensable poner de relieve la importancia de "la materia prima sobre la que la sociedad actual debe aprender del ayer para entender el hoy y comprender el mañana" (Rubio-Muñoz, 2019).

Llegados a es te pun to, nos remitirmos al título de nues tro trabajo y retomamos el "porqué" de la enseñanza de la historia, recuperando la cita de los maestros Joaquin Prats y Jo an S antacana, y a q ue ést a " no de be co ncebirse co mo u n cu erpo de conocimientos acabados, si no co mo una apr oximación al co nocimiento en construcción" (2011: 18). Los adolescentes rechazan el aprendizaje de la historia 
porque se sienten excluidos de ésta, les resulta ajena y poco útil y el discurso histórico resulta muy difícil de comprender (a menudo impregnado de justificantes ideológicos), por lo que al final, acaba siendo un corpus teórico que debe ser memorizado (Santacana, 2002: 88):

Entre las principales causas de dicho rechazo, encontramos:

a) Falta de ent endimiento de los procesos históricos por los propios docentes en formación.

b) Los alumnos de Secundaria suelen tener problemas para empatizar con los modos de vida del pasado.

c) El corsé del currículum educativo dificulta la innovación en el aula.

d) Falta de empatía del alumnado con respecto a la materia. No encuetran un contenido práctico en la historia.

e) Se obvia el desconocimiento de las fuentes para la construcción de la historia y, mucho más, sobre la cimentación del pensamiento histórico.

Respecto a este último punto, las primeras investigaciones sobre la enseñanza de la Historia en torno a los archivos tenían un carácter disperso al terminar el siglo XX, con precedentes en torno a grupos de investigación surgidos a finales de los setenta. Fue el ca so de $\mathrm{G}$ ermanía-75, en la I ínea de I a h istoriografía m arxista como p rimera experiencia sistemática difundida en la docencia, y posteriormente, la de Historia 13-16 siguiendo la New History (Prats, 1989, pp. 201-210 y Sallés, 2010, pp. 5, 10), que propugnaban i mitar en las aulas la metodología del hi storiador, lo más si milar a la praxis real. Ya a finales de los noventa fueron surgiendo otras reflexiones generales sobre el tema, tanto en el ámbito europeo (Walne, 1985), como en el marco español (Estepa, 1995: 55-72 y 2004: 33-46).

Para construir un conocimiento activo mediante el aprendizaje por descubrimiento y el uso del archivo, la aproximación deberá efectuarse mediante caminos que permitan al alumnado de S ecundaria indagar; aproximarse al hech o hi storico, ver, tocar y se ntir sus fuentes y comprender el método de acceso a éstas (Prats y Santacana, 2011: 18). Aún queda mucho personal docente reacio a enseñar investigando, como estrategia 
básica par a el apr endizaje de I a hi storia. $Y$, efectivamente, no só lo se t rata de averiguar qué fuentes debemos estudiar o cuáles resultan más adecuadas para un ejercicio educativo eficaz que posibilite la interacción entre los fondos archivísticos y la enseñanza de la hi storia ( Tribó, 2002), sino que hem os de di señar propuestas de acción di dáctica. Para iniciar a I os adolescentes en est e nuevo m odo de estudiar la historia es necesario, además de acceder al archivo, crear materiales educativos y ofrecer pautas previas sobre cómo empezar a trabajar en di chas instituciones. Debe existir una I abor d idáctica preliminar por par te del doce nte, que i mplica una i ngente tarea de documentación, estudio y preparación de materiales y documentos que serán objeto de análisis, así como verificar su conexión con la temática que se trabajará en el aula antes, durante y después de la visita al archivo. Todo ello exige un compromiso previo, gran motivación y dispensar enormes cantidades de tiempo, en g ran medida robado al ocio o a la familia.

La Orden de 15 de enero de 2021, por la que se desarrolla el currículo correspondiente a I a et apa de E ducación S ecundaria Obligatoria en I a C omunidad Autónoma de Andalucía, est ablece I a nece sidad de su brayar I as conexiones entre pasa do y presente, incentivando la comprensión del cambio y la continuidad en las estructuras sociales, efectuando un análisis más allá del simple aprendizaje memorístico diacrónico y si ncrónico de la hi storia. A simismo, I a nor ma plasma la nece sidad de reconocer los derechos c ívico-políticos y socio-económicos para el ejercicio de una ciudadanía democrática, haciendo hincapié en el crecimiento y desarrollo económicos de Andalucía en la historia, aproximación a los cambios tecnológicos, unidos a los conflictos socioeconómicos derivados de estos, así como a l os mecanismos para su prevención y/o resolución.

Por ot ra parte, la LOMLOE (Ley Orgánica 3/2020, de 29 de di ciembre, por la que se modifica la Ley Orgánica 2/2006, de 3 de m ayo, de E ducación), destina la franja de cursos de $1^{\circ}$ a $3^{\circ}$ de I a E SO para la impartición de I a asg inatura de G eografía e Historia (Artículo 24.e), y el curriculum andaluz incita a la conexión del aprendizaje de la historia con algunas problemáticas sociales relevantes, conectando la materia con otras de la etapa, como Economía, Educación para la Ciudadanía o Iniciación a la Actividad Emprendedora y Empresarial. Se vincula así nuestra propuesta didáctica: Ia crisis económica de 1917 en M álaga y su apl icación al presente a I os contenidos 
curriculares del $3^{\circ}$ curso de la ESO, dentro del Bloque 2: El espacio humano, abordándose el espacio geográfico andaluz en el sistema productivo mundial; los sistemas y se ctores económicos; I a i nclusión social; el desa rrollo eco nómico; la sostenibilidad $\mathrm{m}$ edioambiental y la buena $\mathrm{g}$ obernanza; los principales espacios económicos andaluces y la organización política de las sociedades, entre otras cuestiones.

Sostenemos que la E ducación Secundaria Obligatoria constituye la et apa educa tiva idónea par a transmitir v alores al al umnado mediante la ense ñanza de una hi storia holística, que co mprenda el em pleo de I a m etodología pr opia del hi storiador co mo elemento esencial en la construcción de la misma, y sobre todo, que conciba la utilidad del pasa do par a l a co mprensión del pr esente, m ediante l a co rrecta i niciación a la cimentación del pensa miento histórico. Así pues, el cu rrículo andal uz e stablece que mediante la enseñanza de la materia Geografía e Historia la ESO el alumnado deberá adquiririr una se rie de ca pacidades, condensadas en obj etivos, ent rel os que destacamos el siguiente: (...) Objetivo 14. Conocer y manejar el vocabulario y las técnicas de investigación y análisis específicas de las ciencias sociales para el desarrollo de las capacidades de resolución de problemas y comprensión de las problemáticas más relevantes de la sociedad actual, prestando especial atención a las causas de los conflictos bélicos, las manifestaciones de desigualdad social, la discriminación de la mujer, el deterioro medioambiental y cualquier forma de intolerancia (Orden de 15 de ener o de 2021). En l o q ue at añe a I a resolución de problemas y co mprensión de I a so ciedad act ual, co incide netamente con I o q ue establece la LOMLOE, con respecto a la recomendación de cursar en estos tres años alguna materia opt ativa co nfigurada co mo un t rabajo monográfico o un pr oyecto interdisciplinar o de colaboración con un servicio a la comunidad (art. 24.3). Asimismo, se fomentaría a través de este tipo de actividad la comprensión lectora, la expresión oral y escr ita, I a co municación audi ovisual y la co mpetencia di gital, así co mo el fomento del espíritu crítico, entre otras competencias (art.25. 6).

\section{Conclusiones.}

A nivel de contenido, la huelga de 1917 en Málaga fracasó o, dicho de otro modo, tuvo un éxi to bast ante ex iguo. E n pr imer I ugar, no f ue secundada por I a peq ueña 
burguesía, ni por los representantes políticos republicanos. Y en segundo lugar, existió una i mportante debi lidad y $\mathrm{f}$ alta de cohesión y pr eparación del pr oletariado concentrado, que se sintió impotente ante la actitud hostil del ejército y de la policía, bastante num erosa y bi en organizada. Sin embargo, I as continuas movilizaciones obreras no cayeron en saco roto. Leyendo con detenimiento las reuniones del Cabildo Malagueño que se reflejan en las Actas Capitulares posteriores al 13 de agosto, podemos comprobar có mo ex istieron mayores co ncesiones al os obreros en su $\mathrm{s}$ peticiones taras la huelga. Las repercusiones tan desiguales de la débil huel ga que tuvo I ugar en $\mathrm{M}$ álaga, también se $\mathrm{r}$ eflejan en I as Actas Capitulares, pues no es precisamente ex tensa l a información que al ude a di cha huelga, tanto en I os meses previos, como en los posteriores a la misma, mencionando el Cabildo su satisfacción ante la cierta calma de las jornadas. No obstante, la incrementación de los dispositivos de vigilancia también se refleja en los documentos de archivo consultados, pues existió un destacado aumento de los efectivos de policía urbana entre los meses de mayo a septiembre de 1917.

En suma, con respecto a Andalucía, es evidente la escasa notoriedad que tuvo la huelga de 1917, pues la historiografía só lo r efleja i nformación par cial r ecogida en publicaciones fragmentarias y $\mathrm{m}$ uy punt uales. Excepto al gunos amagos que se produjeron en localidades cordobesas, gaditanas y jienenses, el conflicto no prosperó como en Valencia o Madrid, por ejemplo.

De todo lo estudiado, extraemos sufiente información para comparar cómo surgían y se desa rrollaban I as huelgas hace un si glo y cómo suceden en I a a ctualidad. L a escasa bibliografía específica sobre Málaga está elaborada casi exclusivamente por la Dra. $\mathrm{M}^{a}$ Dolores Ramos, quien estudió el tema de un modo magnífico. No obstante, se aconseja una revisión bibliográfica por $p$ arte de exp ertos que quizás pueda arrojar nuevos datos al estado de la cuestión. Quizás sería nece sario además, efectuar un análisis en profundidad de los principales sucesos acontecidos en cada provincia andaluza y compararlos con otras regiones españolas, lo que permitiría extraer conclusiones más certeras con respecto a nuestra comunidad autónoma.

A nivel metodológico, hemos puesto de manifiesto que un objetivo claro para un aprendizaje de ca lidad de $\mathrm{I}$ a $\mathrm{h}$ istoria en el cu rrículo de $\mathrm{E}$ ducación $\mathrm{S}$ ecundaria es entender los fenómenos tenidos lugar sobre el t erritorio próximo y co mprender el 
funcionamiento de I as comunidades humanas en el presente y el pasado. El ú nico modo óp timo para c omprender I as sociedades del pasa do es $r$ ealizando comparaciones co $\mathrm{n}$ el presente, y m ucho más efectivo se rá, si nos nut rimos del entorno local, de la sociedad local y del archivo local. En definitiva, la acción didáctica será mucho más fructífera si atendemos a lo local, a lo cotidiano y a lo reconocible, pues es ahí donde el al umnado adol escente puede v erse reflejado. Asimismo, e sta actividad puede proporcionar al alumnado una idea aproximada de cómo se recogía y plasmaba la información en el pasa do, y cómo se recoge en el presente, a raíz del análisis de I os documentos de pr ensa y de las fichas catalográficas de a rchivo elaboradas.

En I a sociedad globalizada act ual, em inentemente "esclava" la i nformación, la enseñanza de la Historia, así como de la construcción del pensamiento histórico, debe ser una premisa básica par a formar a los futuros docentes, pues en el proceso de edificación del pasa do se trasciende de l os espacios y uso $s$ tradicionales de las fuentes, generando con ello una nueva cultura histórica. No cabe la menor duda de la potencialidad educativa de las fuentes primarias para el aprendizaje de la historia, sin embargo, debemos fomentar en las aulas universitarias un modelo de profesor que "investigue al enseñar" y que "enseñe a investigar" a su alumnado, solo así se podrá acercar la historia a los adolescentes sin que produzca rechazo.

Para co ncluir, I as investigaciones de i nnovación doce nte enfocadas a los usos didácticos de los archivos locales españoles se pueden efectuar desde un tratamiento dual, es decir, desd el a pr opia i nstitución y desde las propias fuentes (material documental conservado en ella). Así pues, los archivos albergan óptimas posibilidades pedagógicas, esp ecialmente los locales, porque permiten profundizar en i nformación valiosísima acerca del lugar de residencia del alumnado, ello le permitirá reconocerse en I as fuentes que r ecogen una i nformación que no I e r esulta a jena. Permiten e I conocimiento del patrimonio documental conservado, lo que conducirá a su respeto y valoración.

Utilizar internet, un recurso cotidiano en I a vida de I os adolescentes, como punto de partida en I a búsqueda archivística, les permitirá iniciarse previamente en el rastreo archivístico de Google Académico, Dialnet, imágenes, bases de datos universitarias, y científicas, Censo-Guía de Archivos y/o PARES, etc. Ello posibilitará efectuar a 
posteriori una "visita consciente" del archivo, consultar los instrumentos de descripción y documentación conservada, hacer fichas de documentos, y sobre todo: ver, tocar, oler y sentir el patrimonio archivístico.

\section{Agradecimientos.}

Agradezco la colaboración de los técnicos del Archivo Histórico Municipal de Málaga su disponibilidad y gentileza en este proceso de investigación. Asimismo, agradezco a los coordinadores de los siguientes proyectos, de los cuales soy miembro, los medios necesarios para la publicación de este artículo y en cuyas acciones se enmarca: PIE19-036: Implementación de Mejoras en el proceso de enseñanza-aprendizaje de la Educación Patrimonial en Humanidades, Ciencias Sociales y Educación, 2019-2021 (Coordinadora Dra. M ercedes Fernández $\mathrm{P}$ aradas); Erasmus + CRxSiSS Project: Comparando Recursos para asignaturas en situaciones de gravedad / Comparing Resources for Subjects in Severe Situations (Coordinador Dr. Jesús Delgado Peña).

\section{Referencias}

Campos, M. C. (1998). Género y formación de capital humano: iniciativas públicas y privadas en Málaga durante la crisis de la Restauración, en Trabajo: Revista andaluza de relaciones laborales, $n^{\circ}$ 5-6, (Ejemplar dedicado a: El mercado de trabajo en Andalucía), $97-114$

Chinarro, O. (2018). Cien años de la huelga de 1917. Editorial Pablo Iglesias.

De la torre, H. (2014). Los dictámenes de las repúblicas vecinas. Aportes: Revista de historia contemporánea, año $n^{\circ} 29, n^{\circ} 85,51-81$.

De Lara, J. C.; Domínguez, R. M. y Peña, M. A. (1990): Huelva y la crisis de 1917. Huelva en su historia, 3, 449-504. http://rabida.uhu.es/dspace/bitstream/handle/10272/ 2919/b15131774.pdf sequence $=1$

Diario El Popular, 26/07/1917

Diario El Popular, 19-12-1917.

Diario El Regional, 11-08-1917.

Estepa, J. (1995). El archivo en la enseñanza de la Historia. Tria, 2, 53-72. 
Estepa, J. (2004). El patrimonio documental y los archivos como recursos en la enseñanza de las CC.SS. Rey de las Peñas, R. VII Jornadas archivísticas. Aprender y enseñar con el archivo. (pp. 33-45). Dirección Provincial de Huelva.

Fernández, A. y González Marzo, F. (2003). Uso didáctico del legado histórico conservado en los archivos. El Patrimonio y la Didáctica de las Ciencias Sociales. Asociación de Profesores de Didáctica de las Ciencias Sociales, 553-566.

Florido, A. (2016). El archivo y la es cuela: propuestas didácticas. Boletín ANABAD. LXVI, 2, abril-junio, 2444-7293. https://www.anabad.org/category/publicacionesanabad/boletin/page/2/

Forcadell, C. (1993). De la huelga general al golpe militar: el protagonismo sindical en la crisis de 1917 a 1923. Historia $16, n^{\circ} 201,20-28$.

Fortes, J. A. (1977). España, 1917: la Crisis de Agosto. La s ubversión llega a I os cuarteles. Historia 16, n 16, 89-95.

García, J. (1993). Una mirada ocasional a la evolución histórica del derecho de huelga: el Real Decreto de 23 de marzo de 1917 sobre los conflictos colectivos del trabajo en compañías concesionarias des ervicios públicos. Revista de la Facultad de Derecho de la Universidad Complutense, 17, 39-42.

González, L. y Martín, U. (1995). Los archivos en la enseñanza de la historia. Ayuntamiento de la Laguna. Delegación de Cultura y Patrimonio Histórico Artístico.

Grup 13-16 (1983). 13-16 Hacer Historia. Cymys.

Hernández, F. X. y Serrat, N. (2002). Los archivos en la didáctica de las ciencias sociales, íber, 34, Monográfico: Los archivos en la didáctica de las ciencias sociales. Recuperado de: https://www.grao.com/es/producto/archivos-municipales-y-comarcales-y-didactica-de-la$\underline{\text { historia }}$

Hernández, O. (2013). La huelga de 1917 en Madrid. Historia 2.0: Conocimiento Histórico en Clave Digital, 3-6, 109-131. Recuperado de: https://dialnet.unirioja.es/ejemplar/385263

Iturrate, G. (1998). La función pedagógica del archivo. Aplicación didáctica en forma de talleres de hi storia, tecnológicos y audi ovisuales. Boletín del Instituto Andaluz del Patrimonio Histórico. Año VI, 24, septiembre, 95-105.

Lacomba, J.A. (1985). Andalucía en la crisis española de 1917/1918: El caso de Córdoba. Revista de estudios regionales, 14, 277-298.

Lacomba, J.A. (1968). España en 1917. Ensayo morfológico de una crisis histórica. Saitabi, $1968, n^{\circ} 18,145-162$.

Lacomba, J.A. (1970). La crisis española de 1917. Editorial Ciencia Nueva.

Ley Orgánica 3/2020, de 29 de diciembre, por la que se modifica la Ley Orgánica 2/2006, de 3 de mayo, de Educación.

Ley Orgánica 2/2006, de 3 de mayo, de Educación.

Martín, J. (1971). Huelga general de 1917, Zero. 
Martínez, J.A. ( 2002). H uelga ge neral de 1 917, en S AMBRICIO, C . (ed. lit): Enciclopedia Madrid s.XX. (pp.148-149). Ayuntamiento de Madrid.

Montoya, A. (1977). Ideología y lenguaje en las leyes laborales de España: la crisis de 19171923. Escuela Social.

Orden de 15 de enero de 2021, por la que se desarrolla el currículo correspondiente a la etapa de Educación Secundaria O bligatoria en la C omunidad A utónoma de Andalucía, s e regulan determinados aspectos de la atención a la diversidad, se establece la ordenación de la evaluación del proceso de aprendizaje del alumnado y se determina el proceso de tránsito entre distintas etapas educativas.

Pellejero, M. (1988). D ecadencia del viñedo y c risis pob lacional en la Malaga de finales del siglo XIX. Revista de Historia Económica / Journal of Iberian and Latin American Economic History, 6 (3), 593-633.

Pitocco, F. ( 1985). Archivio, s cuola e insegnamento de lla s toria. Rassegna degli Archivi di Stato, Roma, Vol. XLV, 1-2, gennaio-agosto, 21-28.

Plaza, A. (2019). El sindicalismo ferroviario de origen empresarial en España y la lucha de las compañías contra la acción reivindicativa (1913-1920). Tst: Transportes, Servicios y telecomunicaciones, 39, 81-107. https://www.tstrevista.com/sumarios/sumario 39.asp

Prats, J.(1989). Las experiencias didácticas como alternativas al cuestionario oficial: reflexiones críticas sobre las experiencias de "Germanía-75» e «Historia 13- 16». La enseñanza de las Ciencias Sociales. Visor, 201-210.

Prats, J y Santacana, J . ( 2011a) T rabajar c on $f$ uentes $m$ ateriales e $n$ la enseñanza de la Historia. En J. Prats (coord.), Geografía e historia. Investigación, innovación y buenas prácticas. (pp. 11-17). Graó.

Prats, J. y Santacana, J. (2011b). Enseñar a pensar históricamente: la clase como simulación de la investigación h istórica. Didáctica de la geografía y la historia. Graó/Ministerio de Educación, 67-87.

Ramos, $M^{a}$ D. (1980). Conflictividad s ocial en la c oyuntura de la Primera Guerra Mun dial: Ia huelga de Altos Hornos de Málaga (1916). Baética: Estudios de arte, geografía e historia, 3,303-315.

Ramos, Ma D. (1983). Crisis de subsistencias y conflictividad social en Málaga: Los sucesos de enero de 1918. Baética: Estudios de arte, geografía e historia, 6, 441-466.

Ramos, Mª D. (1987). La Crisis de 1917 en Málaga. Diputación Provincial.

Rey, R. (Dir.). (2004). Aprender y enseñar con el archivo. Diputación Provincial.

Rodriguez-Illera, J. L. y Kaechele, M. (2009). Digital Literacy and Activity Systems in Adolescents. Comparative Information Technology. Springer, 89-102.

Romanelli, F.C. (2000). Archives and educational activities: debate and initiatives in the Italian archives. Archivum, XLV, 267-284.

Rubio-Muñoz, F . J . ( 2019). La $v$ isibilidad d e I o cotidiano. D idáctica, H istoria y $f$ uentes documentales $\mathrm{p}$ ara e l es tudio de la vida u niversitaria en la Salamanca m oderna. El Futuro del Pasado, 10, 373- 392.http://dx.doi.org/10.14516/fdp.2019.010.001.014. 
Sánchez-Campins, C. (2012). Agosto de 1917, primera huelga general indefinida. Madrid histórico, 42, 48-53.

Serrallonga, J. (1991). Motines y revolución. España, 1917. Ayer, 4, (Ejemplar dedicado a: La huelga general), 169-192.

Saborit, A. (1967). La huelga de agosto de 1917: (apuntes históricos). Ed. Pablo Iglesias.

Sallés, N. (2011). La enseñanza de la historia a través del aprendizaje por descubrimiento: evolución del proyecto treinta años después. Enseñanza de las ciencias sociales: revista de investigación, 3-10. https://raco.cat/index.php/EnsenanzaCS/issue/view/18497

Santacana, J. (2002). La investigación en archivo: pautas y propuestas para la escuela. íber, 34, Monográfico: Los archivos en la didáctica de las ciencias sociales. Recuperado de: https://www.grao.com/es/producto/archivos-municipales-y-comarcales-y-didactica-de-la$\underline{\underline{\text { historia }}}$

Suárez, M. (2002). La crisis de 1917. Síntesis.

Tribó, G. (2006). Enseñar a pensar históricamente. Los archivos y las fuentes documentales en la enseñanza de la historia. ICE UB/Horsori.

Tribó, G. (2002). Archivos municipales y comarcales y didáctica de la historia. íber, 34, Monográfico: Los archivos en la didáctica de las ciencias socials. Recuperado de: https://www.grao.com/es/producto/archivos-municipales-y-comarcales-y-didactica-de-lahistoria

Tribó I Traveria, G. (2001). Archivos, fuentes y didáctica de la historia. Historia, antropología y fuentes orales, $25,159-176$.

Tuñón, M. (1974). España, 1917: la Crisis de Agosto. "Agosto sangriento". Historia 16, 16, $72-$ 81.

Tuñón, M. (1981). La dictadura del proletariado: 1917-1920, una crisis institucional. Prensa Periódica.

Tuñón, M. (1978). Luchas obreras y campesinas en la Andalucía del siglo XX: Jaén (19171920), Sevilla (1930-1932). Siglo Veintiuno de España.

Vela, S. (2002). Archivos y Didáctica: un estado de la cuestión. Íber: Didáctica de las Ciencias Sociales, Geografía e Historia. Monográfico: Los archivos en la didáctica de las ciencias sociales, 34, 27-36.

Walne, P. (1985). La administración moderna de archivos y la gestión de documentos: el prontuario Ramp. UNESCO, 381-394.

Pub. bajo licencia internacional CreativeCommons A ttribution-NonCommercial-ShareaAlike 4. 0 (CC-BY-NCSA 4.0). Se permite copiar, usar, distribuir, transmitir y exhibir públicamente, siempre que: i) se reconozca la autoría y la fuente original de publicación (revista, editorial y URL del trabajo); ii) no se utilice con fines comerciales; iii) se mencione la existencia y especificaciones de esta licencia. 